\title{
钯催化端炔的电化学放氢甲酰胺化
}

\author{
程 旭* \\ (南京大学化学化工学院 南京 210023)
}

\section{Palladium-Catalyzed Electrochemical Dehydrogenative Aminocarbonylation of Termianl Alkynes}

\author{
Cheng, $\mathrm{Xu}^{*}$ \\ (School of Chemistry and Chemical Engineering, Nanjing University, Nanjing 210023)
}

一氧化碳是重要的工业气体, 具有合适的氧化态、 稳定性和反应性, 可以向分子中引入羰基, 是一碳化学 的核心原料之一 ${ }^{[1-2]}$. 脱氢甲酰基化反应, 无需预先官能 团化, 实现 $\mathrm{C}-\mathrm{H}$ 底物的直接增羰官能团化, 具有较高 的原子经济性和步骤经济性. 但常见的脱氢甲酰化反应 需要化学计量的氧化剂, 如氧气, 造成可燃助燃气体的 混合, 具有很大的安全风险. 如何实现无氧化剂条件下 的脱氢羰基化反应, 需要通过新的模式来实现 ${ }^{[3]}$.

近来, 武汉大学化学与分子科学学院雷爱文与同事 以端位炔烃为底物和 $\mathrm{CO}$ 为羰基源, 首次实现了钯催化 电化学脱氢甲酰胺化反应 ${ }^{[4]}$.

为了实现相关的转化, 需要克服几个不利因素: (1) 钯的标准电极电势为 $0.92 \mathrm{~V}$ (vs NHE), 采用非隔离池进 行反应, 在阴极表面容易发生 Pd 的还原析出 ${ }^{[5]}$. (2)适用 于 $P d$ 的配体多为膦配体, 存在阳极氧化的可能性, 造成 配合物失活. (3) 一氧化碳与胺在高温下直接生成甲酰 胺, 会干扰炔烃参与的转化 ${ }^{[6-7]}$.

针对以上可能出现的不利因素, 研究人员首先通过 模型底物进行了条件篎选, 对配体、钯前体、溶剂、电 极电势进行了篮选. 在室温和常压下发现了最优条件 (Scheme 1, 中心圆内), 可以 $77 \%$ 的分离收率获得目标 产物 3, 同时氢甲酰胺化产物得到充分地抑制.

为了深入了解配体、溶剂、试剂等对电化学钯催化 反应的影响, 研究人员开展了系统性的循环伏安研究, 发现这些因素可以显著地改变体系中钯物种的氧化还 原电势. 首先, 在阳极 $0.5 \mathrm{~V}(\mathrm{vs} \mathrm{AgCl} / \mathrm{Ag})$ 的电极电势下, 底物胺、炔烃、膦配体和三乙胺都可以很好地耐受, 不 会发生氧化; 而钯物种表现出易于氧化的特性, 膦

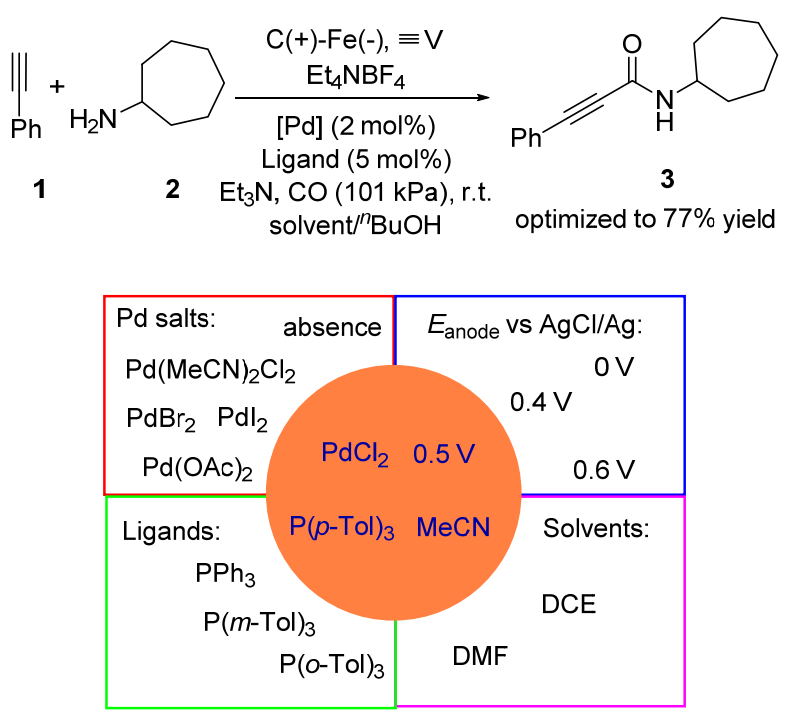

图式 1 电化学放氢甲酰胺化的条件优化

Scheme 1 Optimization of electrochemical aminocarbonylation

配体、溶剂正丁醇都会显著降低钯的氧化电位, 推测是 由于零价钯与富电子配体配位所致. 作为对比, 当 $\mathrm{CO}$ 作为配体时, 零价钯的氧化电位会升高. 另一方面, 测 量钯物种的还原电位时发现, 溶剂、配体都可以稳定二 价钯络合物, 使其不会在实际反应的阴极电极电势范围 内被还原, 确保了二价钯的电化学存活. 这些结果说明, 配体、溶剂、试剂都对钯物种的氧化与还原性质密切相 关, 综合效果使得过渡金属催化和电化学条件契合(图 1).

在此基础上, 研究人员利用原位红外技术对反应的 动力学进行了探究. 在一个接近标准反应条件的模型反 应中, 一氧化碳的扩散, 炔烃和胺的初始浓度对反应速

* Corresponding author. E-mail: chengxu@nju.edu.cn. Published online June 10, 2020. 


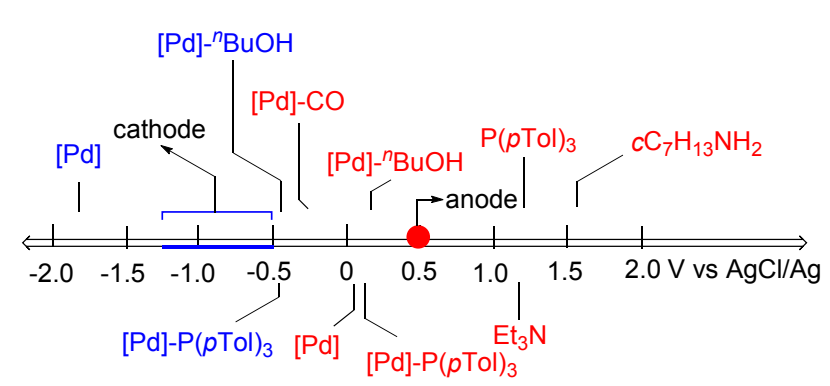

$[\mathrm{Pd}]$ in standard condition: $\mathrm{Pd}\left(\mathrm{MeCN} \mathrm{Cl}_{2}, \mathrm{P}(p \mathrm{Tol})_{3}\right.$, $\mathrm{Et}_{4} \mathrm{NBF}_{4}, \mathrm{MeCN} /{ }^{n} \mathrm{BuOH}, \mathrm{CO}$

The $[\mathrm{Pd}]$ in $\mathrm{CV}$ analysis appears as either $\left[\mathrm{Pd}^{2+}\right]$ in 0 to $-2.0 \mathrm{~V}$ or $\left[\mathrm{Pd}^{0}\right]$ in 0 to $2.0 \mathrm{~V}$

图 1 反应中各物种对钯络合物氧化还原电位的影响

Figure 1 Impact of redox properties of Pd complex by various species in reaction

率影响不大; 而催化剂前体浓度、电极与溶液的接触面 积等因素对反应速率为正相关. 而同位素动力学实验发 现, 底物端炔 $\mathbf{1}$ 中 $\mathrm{C}_{\mathrm{sp}}-\mathrm{H}$ 与其気代物种的 $k_{\mathrm{H}} / k_{\mathrm{D}}=1.21$, 说明端位 $\mathrm{C}-\mathrm{H}$ 键的断裂不是速率控制步骤. 综合这些 实验结果, 研究人员推测电极对 Pd 催化剂的氧化有可 能是反应的速控步(Scheme 2).

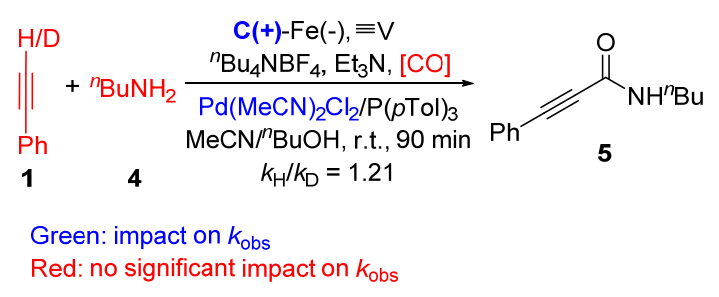

图式 2 反应物种对反应速率的影响 Scheme 2 Impact of factor in reaction on $k_{\mathrm{obs}}$

之后, 研究人员利用快速扫描傅里叶变换 $X$ 射线吸 收精细结构谱(FT-EXAFS)对类似的反应溶液进行了研 究. 测量数据和模拟数据的拟合支持反应中存在 $\mathrm{Pd}^{2+}$ 和 $\mathrm{Pd}^{0}$ 物种(图 2), 通过和离线实验对照证明, 反应初始阶 段 $\mathrm{Pd}^{2+}$ 先和烷基胺配位形成中间体. 进一步, 研究人员 制备了两种可能的二价钯络合物中间体, 在无铜催化剂 条件下炔基钯物种 $\mathbf{6}$ 难以形成(Scheme 3a); 酰基配位的 钯络合物 7 能够顺利地得到目标产物 8 (Scheme 3b). 基 于以上的实验现象, 研究人员提出了一条电化学脱氢反 应路径. 反应中, 二价钯络合物 $\mathbf{A}$ 首先在碱作用下与一 氧化碳和胺生成酰基配合物 $\mathbf{B}$, 进一步与炔发生配位交 换生成 $\mathbf{C}$, 还原消除后变为零价钯 $\mathbf{D}$. 零价钯 $\mathbf{D}$ 在阳极 失去电子恢复到二价钯. 在阴极则发生析氢反应 (Scheme 3c).

研究人员利用这个策略, 以多种端炔为底物, 以铵

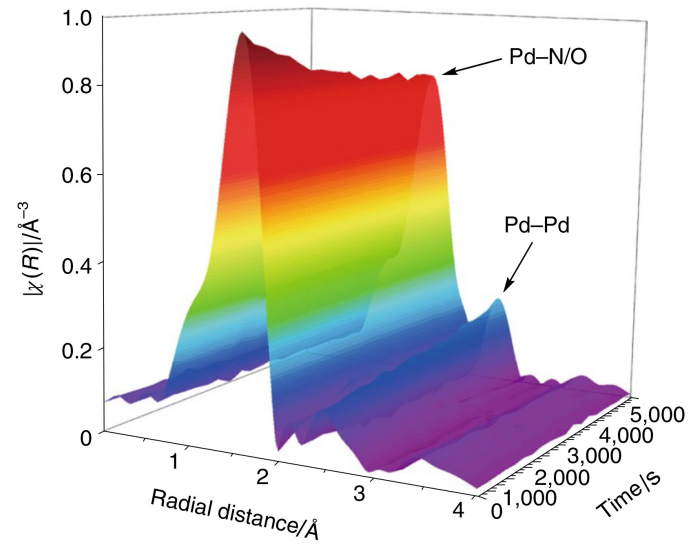

图 2 FT-EXAFS 谱

Figure 2 FT-EXAFS spectra

(a)

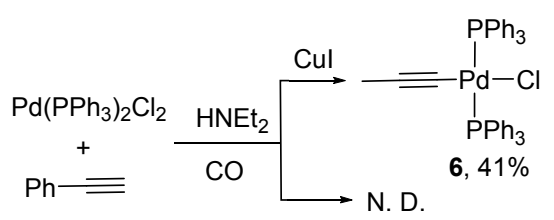

(b)

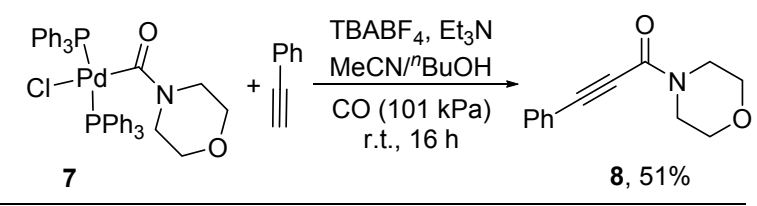

(c)

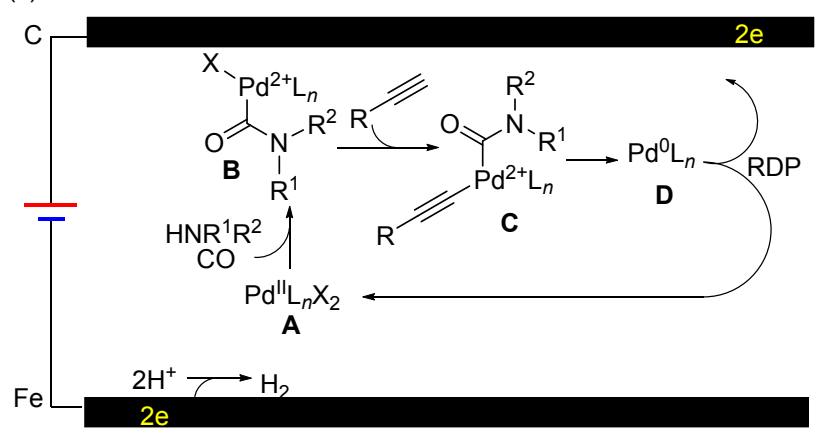

图式 3 可能的关键中间体和反应路径

Scheme 3 Plausible key intermediates and pathway

盐、伯胺、仲胺为氨源, 在一个大气压的一氧化碳氛围 下, 实现多种 $\alpha, \beta$-不饱和炔酰胺的合成, 反应无需任何 外加氧化剂, 直接利用阴极放氢的方式构建目标产物 (Scheme 4).

该工作为电化学条件下钯催化的实验设计提供一 条思路, 为研究电/过渡金属催化中诸多因素的影响提 供了一套方案, 对电化学 $\mathrm{CO}$ 化学的发展提供了一条路 径. 


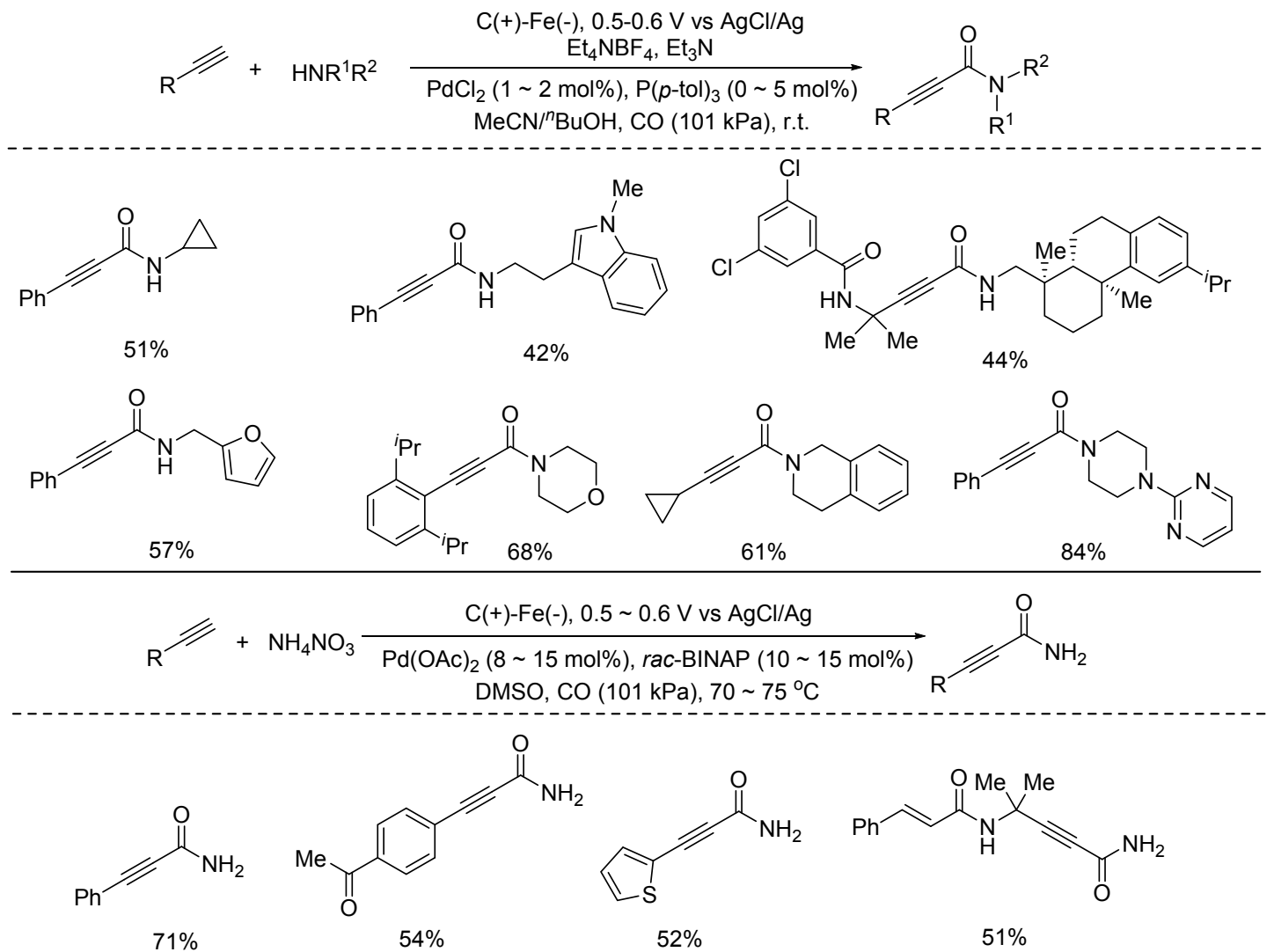

图式 4 底物范围探索

Scheme 4 Exploration of substrate scope.

\section{References}

[1] Barnard, C. F. J. Organometallics 2008, 27, 5402.

[2] Shen, C.; Wu, X.-F. Chem.-Eur. J. 2017, 23, 2973.

[3] Hughes, N. L.; Brown, C. L.; Irwin, A. A.; Cao, Q.; Muldoon, M. J. ChemSusChem 2017, 10, 675.

[4] Zeng, L.; Li, H.; Hu, J.; Zhang, D.; Hu, J.; Peng, P.; Wang, S.; Shi, R.; Peng, J.; Pao, C.-W.; Chen, J.-L.; Lee, J.-F.; Zhang, H.; Chen, Y.-H.; Lei, A. Nat. Catal. 2020, 3, 438.
[5] Speight, J. G. Lange's Handbook of Chemistry, Vol. 1, 16th ed., McGraw-Hill Education, New York, 2005.

[6] Gadge, S. T.; Khedkar, M. V.; Lanke, S. R.; Bhanage, B. M. Adv. Synth. Catal. 2012, 354, 2049.

[7] Zhang, C.; Liu, J.; Xia, C. Catal. Sci. Technol. 2015, 5, 4750.

[8] Yuan, N.; Pascanu, V.; Huang, Z.; Valiente, A.; Heidenreich, N.; Leubner, S.; Inge, A. K.; Gaar, J.; Stock, N.; Persson, I.; Martín-Matute, B.; Zou, X. J. Am. Chem. Soc. 2018, 140, 8206. 